\title{
A REAL-TIME MECHANICAL STRUCTURES MONITORING SYSTEM BASED ON DIGITAL TWIN, IOT AND AUGMENTED REALITY
}

\author{
Roberto Revetria \\ Flavio Tonelli \\ Lorenzo Damiani \\ Department of Mechanical Engineering \\ Genoa University \\ Via Opera Pia 15, 16145 \\ Genoa, ITALY \\ \{Roberto.Revetria, Lorenzo.Damiani, \\ Flavio.Tonelli\}@unige.it
}

\author{
Melissa Demartini \\ Federico Bisio \\ Nicola Peruzzo \\ Department of Mechanical Engineering \\ Genoa University \\ Via Opera Pia 15, 16145 \\ Genoa, ITALY \\ \{ Melissa.demartini, Federico.Bisio, \\ Nicola.Peruzzo\}@dime.unige.it
}

\begin{abstract}
In the Industry 4.0 era, a fundamental role is played by the application of new digital and informatics technologies such as the Digital Twin and the Internet of Things. These technologies offer the possibility of implementing innovative systems related to industrial safety and security. This paper focuses on the application of a new system for monitoring the stresses acting on loaded metal shelving through the use of an integrated application based on augmented reality, the industrial internet of things, digital twin and simulation to improve the safety of the working environment. The stresses are evaluated by the use of strain gauges and a simulation model, the latter being designed to send the strain data to a server. The user has the possibility to access the history of all the stresses during the component lifecycle.
\end{abstract}

Keywords: industry 4.0, digital twin, augmented reality, internet of things, stress analysis.

\section{INTRODUCTION}

In the current competitive scenario manufacturing companies are facing the challenge of digital transformation, as demonstrated by the arrival of Industry 4.0 (Kolberg, 2015). This new paradigm is known as "the fourth industrial revolution" and it refers to new production patterns, including new technologies, productive factors and labor organizations, which are completely changing the production processes and the relationship between customer and company with relevant effects on the supply and value chains (Demartini et al. 2017). Even though most of the aforementioned innovations are in an embryonic stage, they are still an important part of research and progress. The association of these cause new "matched technologies" which could work in a physical and digital environment offering the possibility of improving industrial safety and security among other topics.

This paper offers a particular focus on industrial safety, based on new interconnected technologies (Mladenov et al., 2018) which can highlight risks and dangers within a working environment. The technologies adopted for this scope are Augmented reality (AR), industrial internet of things (IIoT), digital twin and simulation. AR is defined as a real-time direct or indirect view of a physical real-world environment that has been enhanced by adding virtual computer-generated information to it. This technology finds an application in the most diverse situations: from warehouse to production, up to safety and training (Demartini, 2018). AR includes the use of head-mounted displays, virtual retinal displays and smart glasses. The advantages in adopting smart glasses are that they provide information necessary to 
handle a specific situation or work task optimally in real time (Damiani et al. 2018). All wearable devices are connected through the IoT, i.e. the interconnection of informatics devices embedded within everyday objects, allowing the sending and receiving of data. These technologies enable Digital twin to converge data between physical and virtual spaces. The common understanding of the digital twin is that it is a virtual representation which reflects the real world through the multiple phases of the product or plant lifecycle. It embraces new technology by solving existing problems and creating safer production. Therefore, the scope of this paper is to develop an integrated application for monitoring the stresses acting on loaded metal shelving allowing operators to gauge the stress status in real time, thanks to appropriate sensors (strain gauges) connected to the AR devices. Diverse hardware/software and sensor components are used in this application along with the design of a simulation model. The logic behind the application is that the operator can access additional information through the use of smart glasses by two sensors attached to the shelving which are linked to a cloud storage. When the operator sees the QR code attached to the shelving, its data is automatically sent to the cloud and the related simulation model (designed with Matlab), which then provides the stress analysis visible to the operator via the smart glasses. This paper is organized as follows: Section 2 describes the developed application, Section 3 Results and Section 4 Conclusions.

\section{APPLICATION DEVELOPMENT}

The application has been developed using strain gauges, i.e. measuring sensors glued to the structure that vary their electrical resistance proportionally to the actual deformation. Resistance variations are detected by a Wheatstone bridge and the values obtained are collected by an Arduino card, programmed on a dedicated software in $\mathrm{C}++$ language, sent to ThingSpeak, a platform for data analysis and elaboration, through a Wi-Fi module. The data received by the strain gauges can be compared to standard and threshold values made available by the operators, who equipped with AR visors, can assess the stress status in real time and have an idea about the history of the stresses to which the component has undergone in its life. The component is univocally identified by a QR code, which provides its main features, making it a part of the Digital Factory through the Internet of Things concept. The glasses used in this project are the F4 glasses. Figure 1 depicts the logic behind the application.
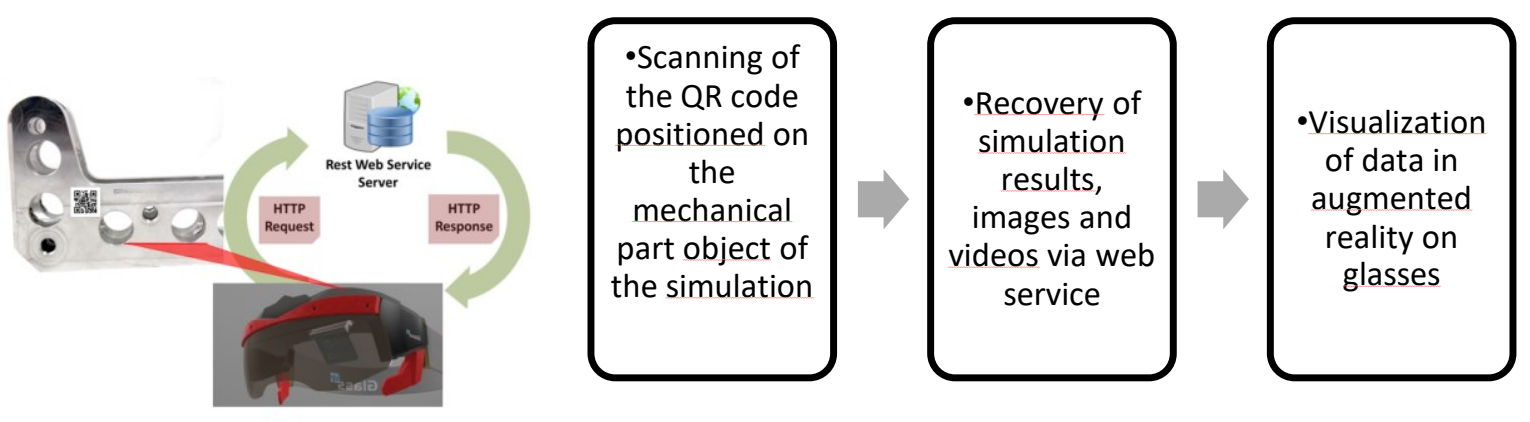

Figure: 1 Logic behind the application.

The hardware components employed for gathering data are:

- Two strain gauges, to assess the shelf deformation and the consequent stresses; the strain gauges were glued to the shelf bracket in the positions indicated in Figure 2;

- Electrical resistors, employed in the Wheatstone bridge, a transducer for detecting the resistance variation of the strain gauges as a voltage across the bridge;

- HX711 amplifier, which measures the voltage values that are too small to be detected by the card; 
- Rapid prototyping card Arduino Mega2560;

- Wi-Fi module ESP82666 to send the data to the ThingSpeak server.

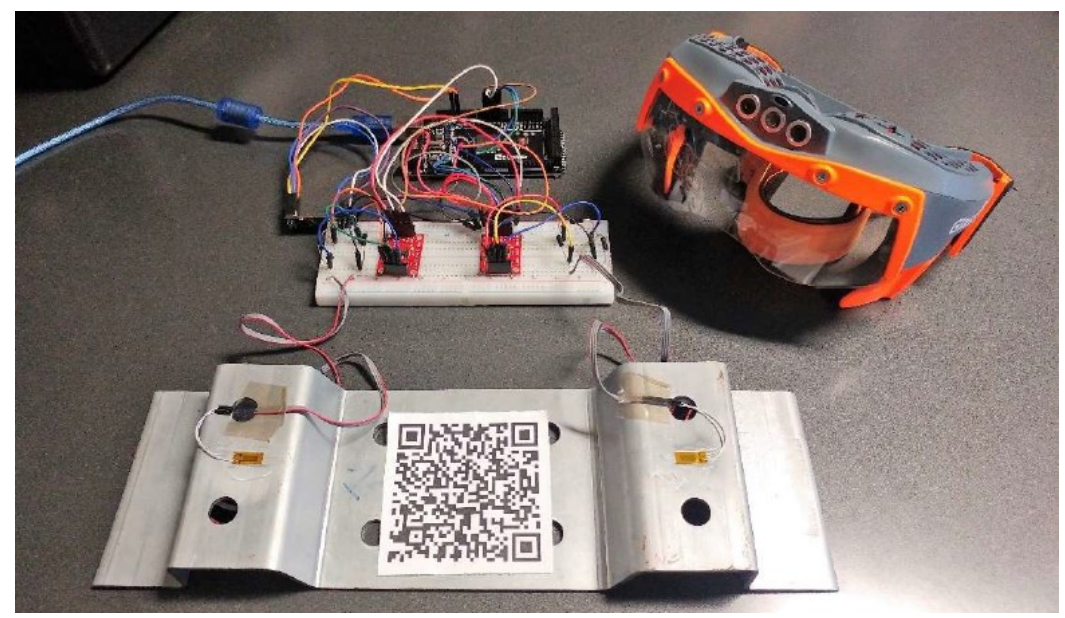

Figure 2: Strain gauges application to the monitored shelf bracket.

The strain gauges operate in coordination with the Wheatstone bridge transducer (see Figure 3), according to the law expressed in Equation 1 (Hoffman, 1989):

$$
\varepsilon=\frac{L-L_{0}}{L_{0}}=\frac{1}{k} \frac{\Delta R}{R_{0}}
$$

$\mathrm{L}$ being the deformed length of the piece, $\mathrm{L}_{0}$ the length at rest, $\mathrm{k}$ the calibration factor of the extensometer, $\Delta \mathrm{R}$ and $\mathrm{R}_{0}$ the extensometer resistance variation and initial resistance respectively.

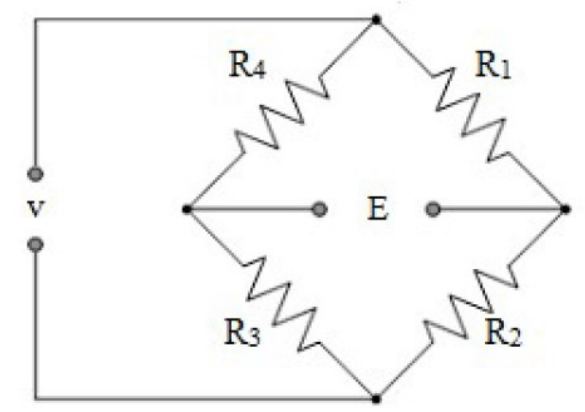

Figure 3: Scheme of a Wheatstone bridge.

Once data is captured through the glasses, it is sent to ThingSpeak through the Wi-Fi module. A specific software has been programmed within the Arduino development environment, using the dedicated library HX711 to analyze the data coming from the two sensors.

\subsection{Software development}

The Arduino card is programmable in a dedicated environment, downloadable for free from the producer's site. The software is available for Windows, Mac and Linux. The Arduino program contained in the 
downloaded folder is an IDE (Integrated Development Environment). The source code of the programs, called sketch, is a simple text file with ino extension. The main elements are:

1) Text editor;

2) Message area;

3) Text console;

4) Tool bar;

5) Menu bar;

6) Status bar.

The basic sketch structure is subdivided in two parts:

- The first part, composed by the setup() function, used to configure the card;

- The second part, composed by the loop() function which contains the program executable in a loop.

Data transmission (Wang et al., 2015) occurs thanks to the ESP8266 router connection, which occurs through AT commands allowing the chip to dialogue with the modem and send data to a server. The data is received by ThingSpeak, the IIoT development platform, which analyzes the data coming from the two sensors. The ThingSpeak basic entity is the channel, each of the variables composing a channel are called fields, a maximum number of 8 fields are allowed per each channel. Two fields, corresponding to the signals of the two strain gauges, have been created, in order to obtain a diagram of the stress in real-time. It is possible to set threshold values that, once exceeded, activate an alarm signal to ensure a fast intervention.

\subsection{MATLAB Finite Element Analysis Toolbox}

This study used the commercial software MATLAB Finite Element Analysis Toolbox (FEA) to simulate the stress state of the metal shelving. This software can provide modeling and simulation of physics, continuum mechanics, and engineering applications with the finite element method. The flow pattern, equivalent stress distribution, equivalent strain distribution, and major and minor strains can all be simulated by FEA. The first step in the modeling process is to create or load the geometry to define the domain to be simulated. Specific data is required such as mechanical and material characteristics, Poisson's ratio (i.e. the negative of the ratio of (signed) transverse strain to (signed) axial strain) and Young's modulus (i.e. a mechanical property that measures the stiffness of a solid material) which create the geometry of the shelving. Due to the structure symmetry, the measurements from half of the geometry structure model is enough to calculate the whole structure. The bracket geometry is shown in Figure 4.

The FEA analysis is carried out online. Since the finite elements model of the shelf is very simple, the computational time required for the parameters calculation does not require more than 5 seconds per each run. 


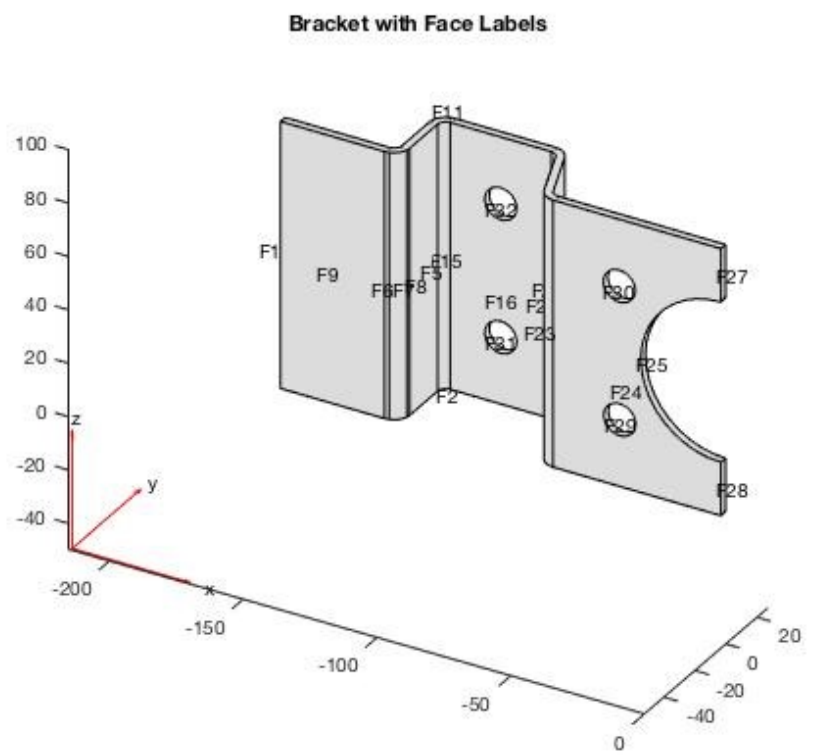

Figure 4: Bracket geometry.

After the bracket geometry has been defined, a computational mesh must be generated to allow for the finite element discretization and thickening of the most critical areas (bends and holes) depicted in Figure 5. Then boundary conditions and constraints are applied:

- REMOTE DESPLACEMENT (strains): used to guide the movement of a face or edge of a geometry from a remote point; it is a linear boundary condition, it responds well to small deformations,

- FIXED SUPPORT: (limits the degrees of freedom) in order to proceed with the analysis and then verify the stress state as a result of the deformations to which the piece is subjected, it is necessary to constrain the model.

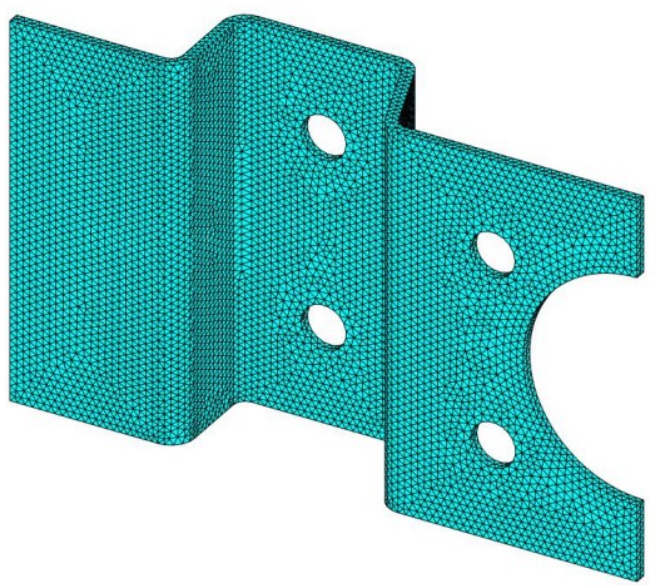

Figure 5: Mesh of the geometry. 
Therefore, the $\mathrm{x}$ and $\mathrm{z}$ displacement are calculated (Figure 6 and 7). At this point it is important to underline that the y displacement has not been calculated because it is minimum and therefore negligible. Finally, Figure 8 describes the equivalent stress.

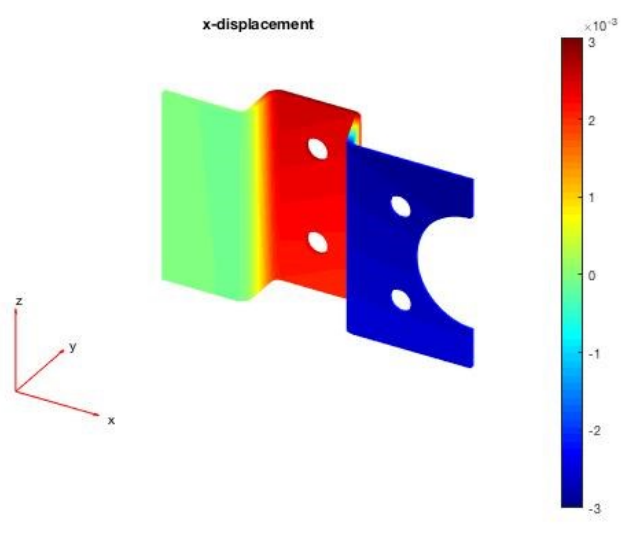

Figure 6: $\mathrm{x}$ displacement.

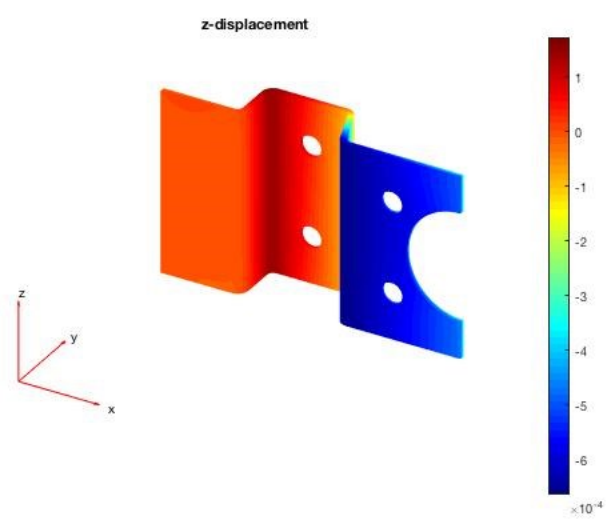

Figure 7: $\mathrm{z}$ displacement.

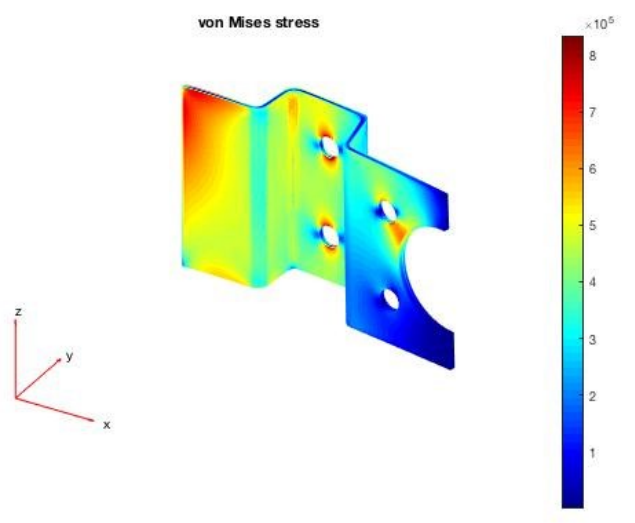

Figure 8: Results of a FEM simulation on a symmetric portion of the shelving. 


\subsection{Calibration}

The shelf has undergone a traction stress test, by means of a traction machine that produces a constant deformation speed to the piece under test conditions. The piece has been stressed by mono-axial stress status, with a displacement of $1 \mathrm{~mm}$ to avoid plastic deformations. By using the traction machine, it made it possible to obtain the instantaneous values of the stress applied to the shelf, together with its displacements under stress and deformation; this data has been used to calibrate the measurement chain. The aim of the tests was to find a calibration factor between the lengthening of the piece and the program exit values. The test has underlined the direct proportionality between lengthening of the piece and output values for both sensors, as visible in Figure 9. The lengthening value measured from calibration has been divided by the initial length and then multiplied by the Young module of the material, to obtain the monoaxial stress. By dividing said stress by the yield value of the material, an exploitation factor, in percentage with respect to the yield stress, has been determined.
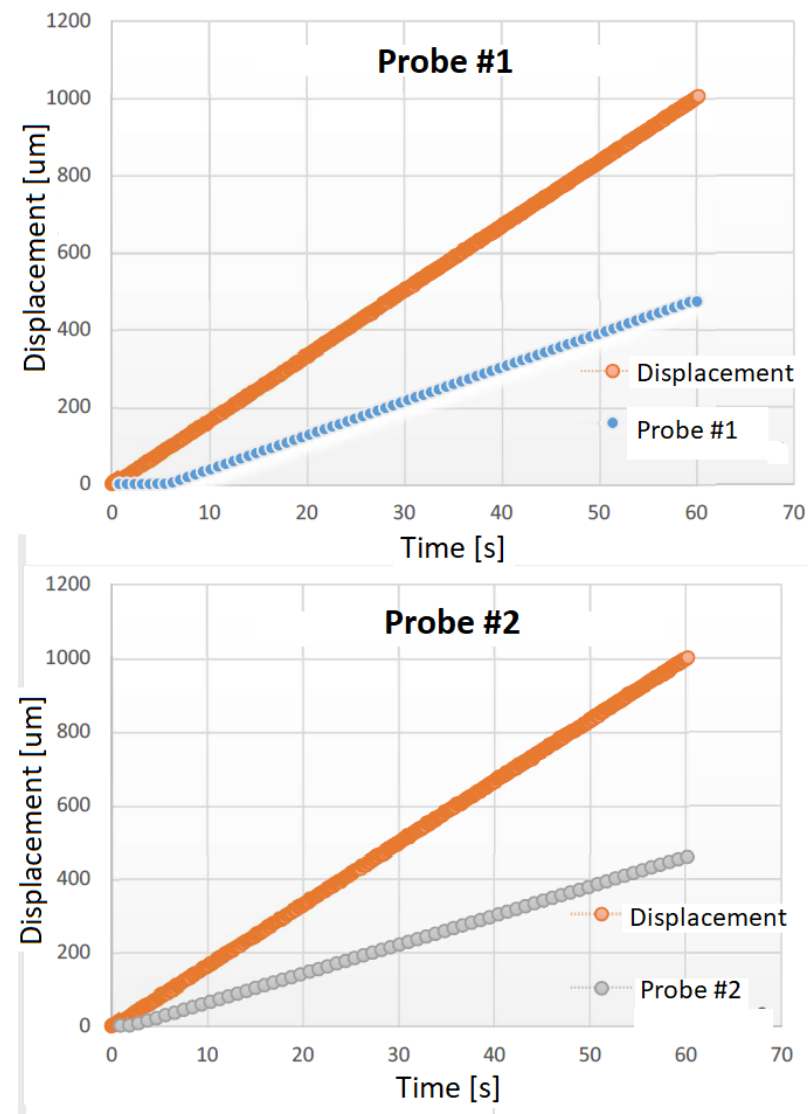

Figure 9: Calibration results for the two strain gauges.

\subsection{Smart glasses}

The glasses used are F4 produced by Glass Up an Italian start-up company. These are equipped with a screen on the right lens. The optical system allows a field of view with an angle of $22^{\circ}$, the effect obtained is the same as a 70" screen positioned at a distance of $5 \mathrm{~m}$. The $3 \mathrm{D}$ view is possible even if the glasses are not binocular. The glasses are equipped with a video-camera which allows video sharing in real time and the data can be visualized through the software "Dashboard". Therefore, the F4 glasses allow both remote assistance and "on the job training" through explicit videos or images. F4 glasses presented in Figure 10. 


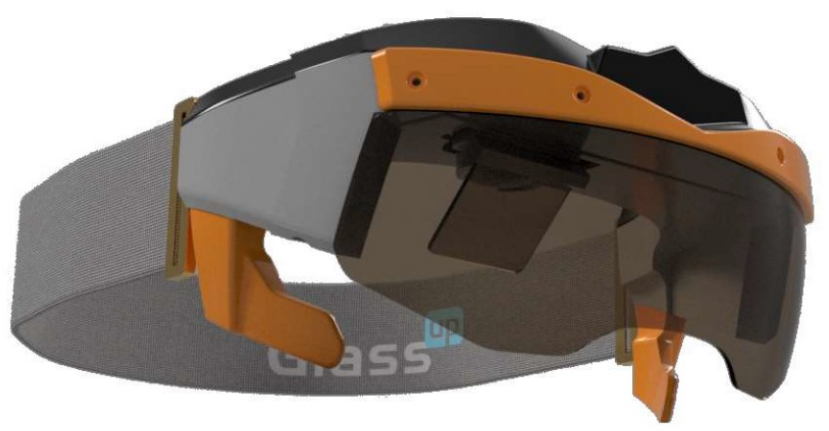

Figure 10: Glass-up F4 augmented reality glasses.

The software can capture an image and re-send it to the user enriched with additional explicit data, text or drawings to improve the information acquisition, or can highlight in real time elements in the field of view to focus the user's attention. Other than the video-camera, other sensors are installed in the F4 glasses: an accelerometer, a compass, a gyroscope, a barometer, a hygrometer, a led torch, plug-in sensors and voice control, all with connections to Wi-Fi, LTE and Bluetooth. Optionally, it is possible to install a $32 \mathrm{X} 32$ thermo-camera or a gas detection sensor. Electric feeding and interaction with the user are ensured by a USB port. The operative system employed is Linux, however a version equipped with Android is under study. The F4 glasses were chosen after a selection process (Mladenov et al., 2018), on the basis of the personalization potentials of its software.

\section{RESULTS}

In this section the results of the experimental activity are discussed. The following picture (Figure 11) represents the diagram of the stress monitoring activity carried out on the shelf. The monitoring has been protracted for 8 minutes and has considered both the sensors installed on the shelving. The diagram represents the Stress Yield Limit [\%] (i.e. the ratio between the measured stress and the yield stress of the material) in function of time. As visible the examined stress values have been recorded analyzing the structure loaded and then unloaded.

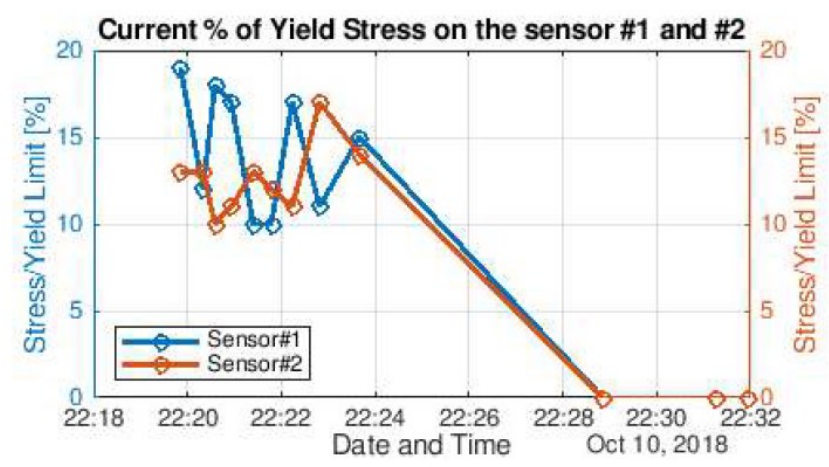

Figure 11: Stress monitoring on the shelving equipped with two strain gauges.

The corresponding parametric calculation program written with MATLAB language can quickly show the stress of mechanical components and the size and location of the maximum stress when the parameters are changed. As stated above, the example test discussed in this paper is a simplification, since the stresses measured are only in the axial direction and calibration has been effected by a traction machine. FEA allows an in-depth analysis of the stress status for a correct positioning of the sensors, and might ensure a precise description of the shelving stress status; starting from a local stress measurement there would be the 
possibility to create a visual representation of the stress status with a chromatic scale, as shown in Figure 8.

\section{CONCLUSIONS}

This paper presented a methodology for monitoring the stress status of metal shelving to be integrated within an AR environment. The system, dedicated to improve safety in the working environment, operational efficiency and product quality, uses strain gauges to assess the stress status of the component and the elaborated results can be visualized by any operator wearing special Virtual Reality glasses. A test has been carried out to assess the potentialities of the system, utilizing a metal shelf loaded with a monoaxial stress as a sample. The study has shown coherent stress values, which have been correctly displayed by the AR visors. The main advantages obtainable by this application are:

- Versatility;

- $\quad$ Ease of employ;

- High data acquisition speed;

- Data intuitiveness;

- Safety and security;

- Alarm function possibilities to indicate excess stress limits;

- Ease of update and upgrade of software.

The advantages of the methodology adopted is the possibility to monitor the stresses in mechanical parts in real time, providing therefore benefits in terms of workers safety and prompt maintenance intervention in case of alert signals.

\section{ACKNOWLEDGMENTS}

The Authors desire to acknowledge the helpful contribution to the development of the study given by prof. Enrico Lertora colleague of DIME dept. Genoa University, who made available his skills and laboratory for the probes calibration.

\section{REFERENCES}

Kolberg D., Zühlk D., 2015, Lean Automation enabled by Industry 4.0 Technologies, IFAC-PapersOnLine 48-3 (2015), pp. 1870-1875.

Demartini, M., Tonelli, F., Damiani, L., Revetria, R., Cassettari, L. Digitalization of manufacturing execution systems: The core technology for realizing future smart factories (2017) Proceedings of the Summer School Francesco Turco, 2017-September, pp. 326-333.

Mladenov B., Damiani L., Giribone P. and Revetria R., A Short Review of the SDKs and Wearable Devices to be Used for AR Application for Industrial Working Environment, Proceedings of the World Congress on Engineering and Computer Science 2018 Vol I, WCECS 2018, October 23-25, 2018, San Francisco, USA, pp. 137-142.

Damiani, L., Demartini, M., Guizzi, G., Revetria, R., Tonelli, F., Augmented and virtual reality applications in industrial systems: A qualitative review towards the industry 4.0 era (2018) IFAC-PapersOnLine, 51 (11), pp. 624-630.

Damiani, L., Revetria, R., Volpe, A. Augmented reality and simulation over distributed platforms to support workers (2016) Proceedings - Winter Simulation Conference, 2016-February, art. no. 7408476, pp. 3214-3215.

Demartini, M., Tonelli, F. Quality management in the industry 4.0 era (2018) Proceedings of the Summer School Francesco Turco, 2018-September, pp. 8-14. 
Hoffmann K., 1989, An introduction to measurements using strain gages, Hottinger Baldwin Messtechnik GmbH, Darmstadt.

J. Wang, L. Zhang, L. Duan, R. Gao, A new paradigm of cloud-based predictive maintenance for intelligent manufacturing, March 2015, Journal of Intelligent Manufacturing, DOI: 10.1007/s10845-015-1066-0.

Revetria, R., Catania, A., Cassettari, L., Guizzi, G., Romano, E., Murino, T., Improta, G., Fujita, H. Improving healthcare using cognitive computing based software: An application in emergency situation (2012) Lecture Notes in Computer Science (including subseries Lecture Notes in Artificial Intelligence and Lecture Notes in Bioinformatics), 7345 LNAI, pp. 477-490.

\section{AUTHOR BIOGRAPHIES}

FEDERICO BISIO is a graduate engineer at Mechanical Engineering Department of Genoa University. His research interests include industrial plants, modeling and simulation. scs19roeder@gmail.com.

NICOLA PERUZZI is a graduate engineer at Mechanical Engineering Department of Genoa University. His research interests include industrial plants, modeling and simulation. His email address is scs19frazier@gmail.com.

LORENZO DAMIANI is a Researcher in the Mechanical Engineering department of Genoa University. He holds a Ph.D. in Engineering from Genoa University, His research interests include industrial plants, modeling and simulation. His email address is Lorenzo.Damiani@unige.it.

MELISSA DEMARTINI is a Researcher in the Mechanical Engineering department of Genoa University. She holds a Ph.D. in Engineering from Genoa University, Her research interests include industrial plants, modeling and simulation. His email address is melissa.demartini@dime.unige.it

ROBERTO REVETRIA is a Full Professor in the Mechanical Engineering Department of Genoa University, received his Ph.D. from Genoa University, His research interests include modeling, simulation and industrial plants. His email address is Revetria@dime.unige.it.

FLAVIO TONELLI is a Full Professor in the Mechanical Engineering Department of Genoa University, received his Ph.D. from Genoa University, His research interests include modeling, simulation and industrial plants. His email address is Flavio.tonelli@unige.it 